\title{
Group Birefringence Measurement in Photonic Liquid Crystal Fibers by Use of Narrowband Light Sources
}

\author{
D. BudaszeWski* And A.W. Domański \\ Faculty of Physics, Warsaw University of Technology \\ Koszykowa 75, 00-662 Warsaw, Poland
}

\begin{abstract}
In the paper we present the results of investigating the effective group birefringence of photonic crystal fiber, partially infiltrated with 6CHBT liquid crystal mixture, which is a chemical abbreviation for 4-(trans-4- $n$ -hexylcyclohexyl)isothiocyanatobenzene. We also introduce a novel method of group birefringence measurement based on phenomenon of depolarization of partially coherent light in birefringent media. We use Mueller-Stokes matrix formalism extended by additional depolarization matrix, which gives us possibility to calculate degree of polarization fluctuations of the light propagating in the liquid-crystal infiltrated microstructured optical fibres. We conducted the research for green and red semiconductor laser diodes. The results may contribute in construction of tunable photonic liquid crystal fibers depolarizer. Additionally, we have controlled birefringence of the photonic liquid crystal fibers by external electric field. These research may lead to designing a new type of fiber optic depolarizer based on photonic liquid crystal fibers.
\end{abstract}

PACS numbers: 42.79.Kr, 42.25.Bs

\section{Introduction}

Over the last two decades there is a great interest in novel combination of photonic crystal fibers $(\mathrm{PCF})$ and liquid crystal (LC) mixtures, also known as photonic liquid crystal fibers (PLCF) [1]. Such structures (Fig. 1) possesses a new uncommon properties, like polarization tuning of light propagating inside the structure.

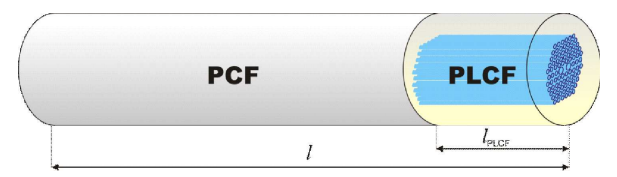

Fig. 1. PCF partially infiltrated with LC mixture forming a PLCF.

In general, state and degree of the light polarization depend on birefringence of the medium. The PLCFs may be highly birefringent and may change their parameters dependent on external parameters such as temperature, electric and optical fields.

In general, partially coherent light is being depolarized by birefringent medium. Both orthogonal components of wave package may have different phase and group velocities, which may result in changes of the state and degree of polarization (SOP and DOP) (Fig. 2).

\footnotetext{
* corresponding author; e-mail: danielb@if.pw.edu.pl
}

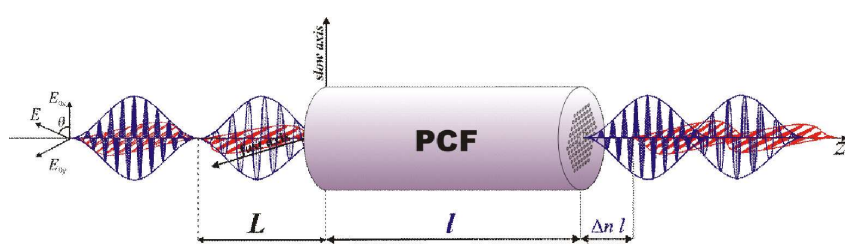

Fig. 2. Phenomenon of depolarization of partially coherent light in birefringent medium. $L$ - length of wave package, $l$ - length of birefringent medium, $\Delta n$ - birefringence of the medium.

The best way to describe degree of polarization changes of light guided through birefringent medium is the Mueller-Stokes matrix formalism. We have extended it by additional matrix, which characterizes depolarization properties of the medium [2].

$$
\begin{aligned}
& {\left[\begin{array}{c}
S_{0}^{\text {out }} \\
S_{1}^{\text {out }} \\
S_{2}^{\text {out }} \\
S_{3}^{\text {out }}
\end{array}\right]=\left[\begin{array}{cccc}
1 & 0 & 0 & 0 \\
0 & P_{\mathrm{C}} & 0 & 0 \\
0 & 0 & P_{\mathrm{C}} & 0 \\
0 & 0 & 0 & P_{\mathrm{C}}
\end{array}\right]} \\
& \times\left[\begin{array}{llll}
m_{11} & m_{12} & m_{13} & m_{14} \\
m_{21} & m_{22} & m_{23} & m_{24} \\
m_{31} & m_{32} & m_{33} & m_{34} \\
m_{41} & m_{42} & m_{43} & m_{44}
\end{array}\right]\left[\begin{array}{c}
S_{0}^{\text {in }} \\
S_{1}^{\text {in }} \\
S_{2}^{\text {in }} \\
S_{3}^{\text {in }}
\end{array}\right],
\end{aligned}
$$

where $\left[S_{x}^{\text {in }}\right]$ and $\left[S_{x}^{\text {out }}\right]$ are parameters of the input and output Stokes vectors, respectively, $m_{i j}$ are elements of 


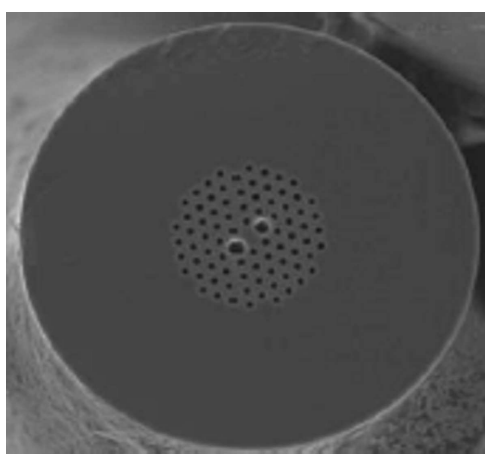

Fig. 3. Blazephotonic PM-1550-01 fiber structure.

the Mueller matrix of the medium and $P_{\mathrm{C}}$ are elements of a depolarization matrix. DOP may be directly calculated from the elements of the Stokes vector, which can be measured by using a quarter-wave plate and an analyzer

$$
\begin{aligned}
& \mathrm{DOP}=\frac{I_{\text {polarized }}}{I_{\text {total }}}=\frac{\sqrt{S_{1}^{2}+S_{2}^{2}+S_{3}^{2}}}{S_{0}},
\end{aligned}
$$

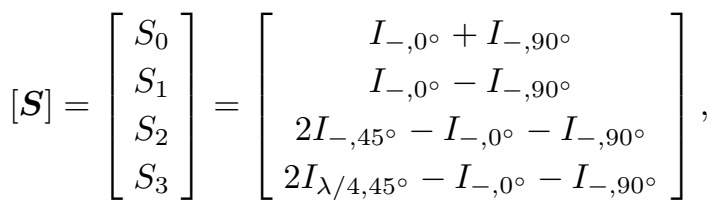

where first subscript defines lack or presence of the quarter-wave plate, and second subscript defines the azimuth of the analyzer.

During the propagation through a birefringent medium the length shift is increasing and the degree of polarization diminishes according to the formulae [3]:

$$
\begin{aligned}
& \mathrm{DOP}=P_{\mathrm{G}} \\
& \quad=\sqrt{1-\left(1-\exp \left(-2\left(\frac{\Delta n l}{\Delta L_{\mathrm{G}}}\right)^{2}\right)\right) \sin ^{2}(2 \theta),} \\
& \mathrm{DOP}=P_{\mathrm{L}} \\
& =\sqrt{1-\left(1-\exp \left(-2 \frac{\Delta n l}{\Delta L_{\mathrm{L}}}\right)\right) \sin ^{2}(2 \theta),}
\end{aligned}
$$

where $l$ and $\Delta n$ are medium length and birefringence, respectively, $\Delta L_{\mathrm{G}, \mathrm{L}}$ is a coherence length of light with Gaussian or Lorentzian spectral distribution, $E_{0 x}, E_{0 y}$ are the electric field components amplitudes of the light, $\theta$ is the azimuth between polarization plane and birefringence axis of the medium.

When the azimuth $\theta$ is of $45^{\circ}$ the degree of polarization reaches its minimum value. Depending on birefringence, path length of the medium and coherence length light beam may be totally depolarized at the output of the medium.

By measuring DOP we are able to calculate absolute values of group birefringence of investigated PLCF.

\section{Depolarization in photonic crystal fibers}

Birefringence in optical fibers is characterized by its beat length $L_{\mathrm{B}}$ :

$$
L_{\mathrm{B}}=\frac{2 \pi}{\left|\beta_{x}-\beta_{y}\right|}=\frac{\bar{\lambda}}{B},
$$

where $\beta_{x}, \beta_{y}$ are propagation constants of both orthogonal polarization modes, $\bar{\lambda}$ means central wavelength of the light source, and $B$ is phase modal birefringence of the fiber. $L_{\mathrm{B}}$ is beat length of the fiber after which the state of polarization is being reconstructed. The formulae for DOP diminishing in birefingent fibers are similar to the anisotropic crystal configuration $[4,5]$ :

$$
\begin{gathered}
P_{\mathrm{L}}=\sqrt{1-\left[1-\exp \left(-2 \eta_{\mathrm{L}}\right)\right] \sin ^{2} 2 \theta}, \quad \text { where } \\
\eta_{\mathrm{L}}=\frac{\bar{\lambda} L}{\Delta L L_{\mathrm{B}}}
\end{gathered}
$$

and

$$
\begin{aligned}
P_{\mathrm{G}} & =\sqrt{1-\left[1-\exp \left(-2 \eta_{\mathrm{G}}\right)\right] \sin ^{2} 2 \theta}, \quad \text { where } \\
\eta_{\mathrm{G}} & =\left(\frac{\bar{\lambda} L}{\Delta L L_{\mathrm{B}}}\right)^{2} .
\end{aligned}
$$

In birefringent media, like PLCF two values of light velocities can be distinguished: phase velocity defined as $v_{\mathrm{p}}=\frac{\omega}{k}$, and group velocity defined as $v_{\mathrm{g}}=\frac{\mathrm{d} \omega}{\mathrm{d} k}$, where $\omega$ is the frequency of light, and $k$ is the wave number. The value of phase birefringence $B$, which depends on the difference between orthogonal polarization modes phase velocities $v_{\mathrm{p}_{x}}$ and $v_{\mathrm{p}_{y}}$, is different from group birefringence value $G$, which depends on the difference between group velocities of the polarization modes $v_{\mathrm{g}_{x}}$ and $v_{\mathrm{g}_{y}}$ :

$$
\begin{aligned}
& B(\lambda)=n_{x}(\lambda)-n_{y}(\lambda)=\frac{c}{v_{\mathrm{p}_{x}}(\lambda)}-\frac{c}{v_{\mathrm{p}_{y}}(\lambda)}, \\
& G(\lambda)=N_{x}(\lambda)-N_{y}(\lambda)=\frac{c}{v_{\mathrm{g}_{x}}(\lambda)}-\frac{c}{v_{\mathrm{g}_{y}}(\lambda)},
\end{aligned}
$$

where $n_{x}$ and $n_{y}$ are wavelength dependent refractive indices for polarization modes, while $N_{x}$ and $N_{y}$ are the group refractive indices, which can be calculated from

$$
N=n-\lambda \frac{\mathrm{d} n}{\mathrm{~d} \lambda} .
$$

Thus the phase birefringence can be defined as:

$$
B=\left|n_{x}-n_{y}\right|=\frac{\left|\beta_{x}-\beta_{y}\right|}{k}
$$

and the group birefringence can be obtained from

$$
G=B-\lambda \frac{\mathrm{d} B}{\mathrm{~d} \lambda}=-\frac{\lambda^{2}}{2 \pi} \frac{\mathrm{d}\left[\frac{B(\lambda)}{\lambda}\right]}{\mathrm{d} \lambda},
$$

where $\beta_{x}$ and $\beta_{y}$ are the propagation constants of two orthogonal polarization modes. If the phase birefringence remains a constant value in broad range of wavelengths (as it does for standard polarization maintaining fibers) it is equal to group birefringence $(G=B)[6]$.

\section{Experimental setup}

The main aim of the research was to deal with group birefringence fluctuation measurement of the PLCF by 
use of novel method based on depolarization of partially temporal coherent light phenomenon.

A commercially available polarization maintaining photonic crystal fiber (type Blazephotonic HB-1550-01) fabricated by CrystalFibre has been partially infiltrated with liquid crystal mixture 6CHBT and put under investigation in described experiment. The fiber has a silica glass core and the light is index-guided due to higher index of refraction in the core than in partially holey cladding. The fiber structure is shown in Fig. 3 and its parameters are presented in Table I.

TABLE I

Blazephotonic PM-1550-01 fiber parameters.

\begin{tabular}{c|c}
\hline \hline Parameters & $\begin{array}{c}\text { Blazephotonic } \\
\text { PM-1550-01 }\end{array}$ \\
\hline large hole diameter $(D)[\mu \mathrm{m}]$ & 4.5 \\
small hole diameter $(d)[\mu \mathrm{m}]$ & 2.2 \\
fibre diameter $[\mu \mathrm{m}]$ & 125
\end{tabular}

The experimental setup for measuring group birefringence of the PCF is shown in Fig. 4.
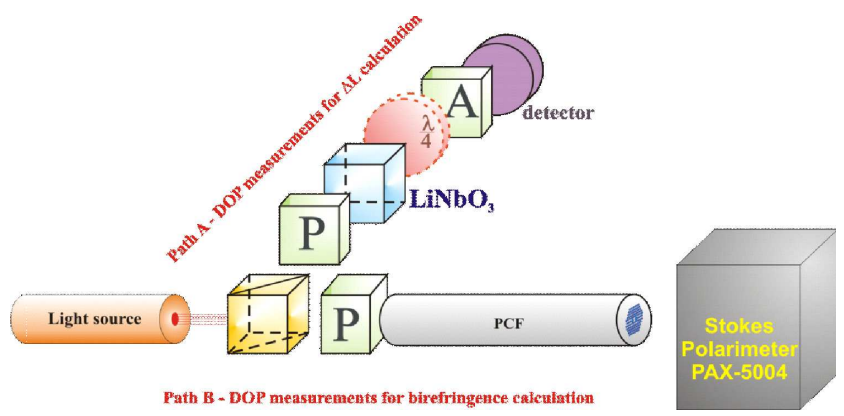

Fig. 4. Experimental setup for measuring DOP fluctuations in PCF.

The partially coherent light beam from laser diode (LD) with Lorentzian spectrum was split by beam splitter and directed into two separate paths. In the path $\mathrm{A}$ the DOP of the light beam was measured after passing through the lithium niobate anisotropic crystal with well known birefringence and path length. To calculate DOP of the narrow-band light sources used in the experiment we used classical method of measuring four intensities of light with different configurations of analyzer and quarter wave plate (Eq. (3)). According to Eq. (5) we are able to calculate the coherence length of the light beams used in the setup

$$
\Delta L_{\mathrm{L}}=-\frac{\left|\Delta n_{\mathrm{LiNbO} 3}\right| l_{\mathrm{LiNbO} 3}}{\ln \left(P_{L_{\mathrm{LiNbO}}}\right)} .
$$

In Fig. 5 we present two spectrograms collected from high precision Optical Spectrum Analyzer ANDO AQ-6315E.

We have calculated the coherence length of these light beams according to the equation $\Delta L_{\mathrm{L}}=\frac{\lambda^{2}}{\Delta \lambda} \frac{1}{\pi}$ and com-

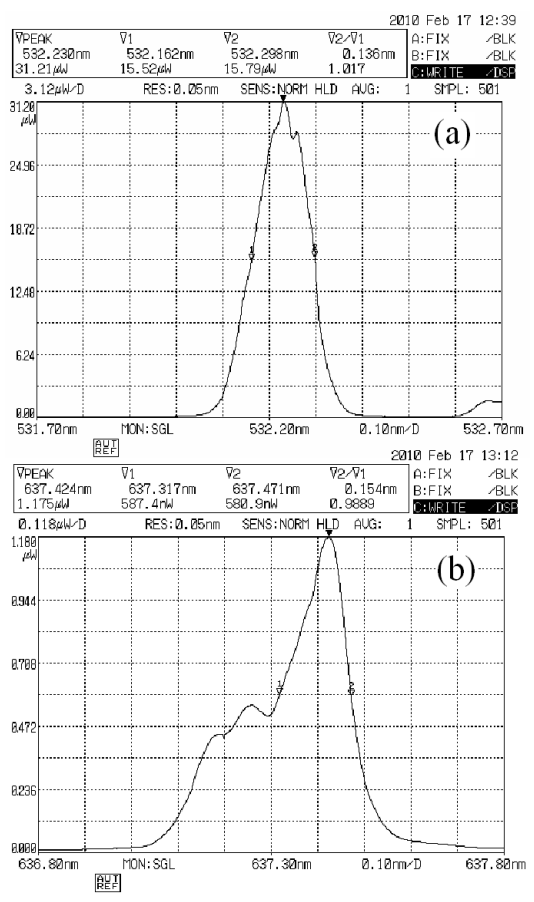

Fig. 5. Spectrograms of two light sources used in the experiment: (a) green LD, (b) red LD. The parameters of the light beams are shown at spectrograms.

pared it with the values obtained from our depolarization method. As shown at Table II the values stand in good agreement.

TABLE II

Comparison of values of coherence length for both light sources used in the experiment obtained by two methods.

\begin{tabular}{c|c|c}
\hline \hline & Green LD & Red LD \\
\hline$\Delta L_{\mathrm{L}}=\frac{\lambda^{2}}{\Delta \lambda} \frac{1}{\pi}$ & $\Delta L_{\mathrm{L}}=\frac{\lambda^{2}}{\Delta \lambda} \frac{1}{\pi}=$ & $\Delta L_{\mathrm{L}}=\frac{\lambda^{2}}{\Delta \lambda} \frac{1}{\pi}=$ \\
& $0.714 \times 10^{-3} \mathrm{~m}$ & $0.839 \times 10^{-3} \mathrm{~m}$ \\
\hline depolarization & $\Delta L_{\mathrm{L}}=$ & $\Delta L_{\mathrm{L}}=$ \\
based method & $0.662 \times 10^{-3} \mathrm{~m}$ & $1.171 \times 10^{-3} \mathrm{~m}$
\end{tabular}

In the path B the group birefringence of the $1 \mathrm{~m}$ long PCF was calculated. Partially coherent light beam was coupled into PCF, and the DOP at the output was measured by use of high precision Stokes polarimeter THORLABS PAX-5710.

From Eq. (16) rewritten for PCF we were able to derive equation describing group birefringence responsible for depolarization phenomenon in investigated PCF:

$$
G=\Delta n_{\mathrm{eff}}=-\frac{\Delta L_{\mathrm{L}} \ln \left(P_{\mathrm{L}_{\mathrm{PCF}}}\right)}{l_{\mathrm{PCF}}} .
$$

It should be pointed out that it is impossible to determine the sign of the birefringence by measuring drop of the DOP, thus only absolute values have been calculated. 
By use of described method we have calculated group birefringence of PCF infiltrated with $\mathrm{LC}$ and placed in the external electric field $[6,7]$. As it was mentioned in the beginning of this Section we have used $1 \mathrm{~m}$ long 1550 Blaze Photonics PCF and partially filled its micro holes with molecules of liquid crystal 6CHBT. We have placed filled part of the PCF in the glass capillary with four electrodes as shown in Fig. 6.

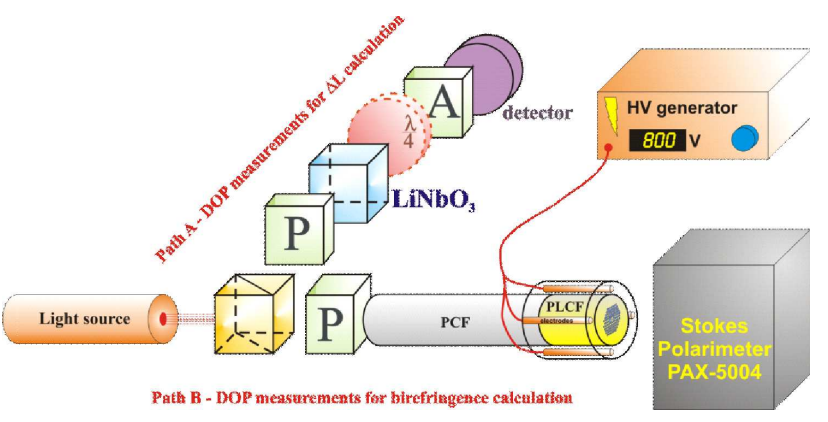

Fig. 6. Experimental setup for measuring group birefringence in the PLCF under external electric field.

The electric field was generated between two opposite electrodes. Under the electric field the molecules of LC were changing their orientations inside the micro-holes, which resulted in changes of effective group birefringence of the PLCF.

\section{Experimental results and analysis}

In the empty part of the PCF light was guided by modified total internal reflection (mTIR) mechanism. In the filled part of the PCF the refractive index of the core was smaller than effective refractive index of the cladding, hence light was guided by the photonic bandgap mechanism (PBG). In our experiment both laser diodes were operating in the transmission range, as shown in Fig. 7.

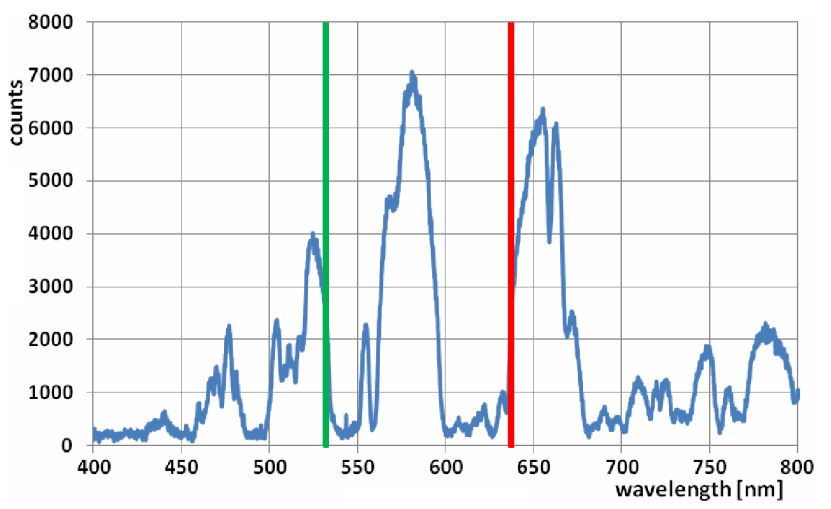

Fig. 7. Transmission spectrum of the PLCF and two wavelengths used in the research.

We have applied external electric field from function generator connected to the high voltage amplifier. By
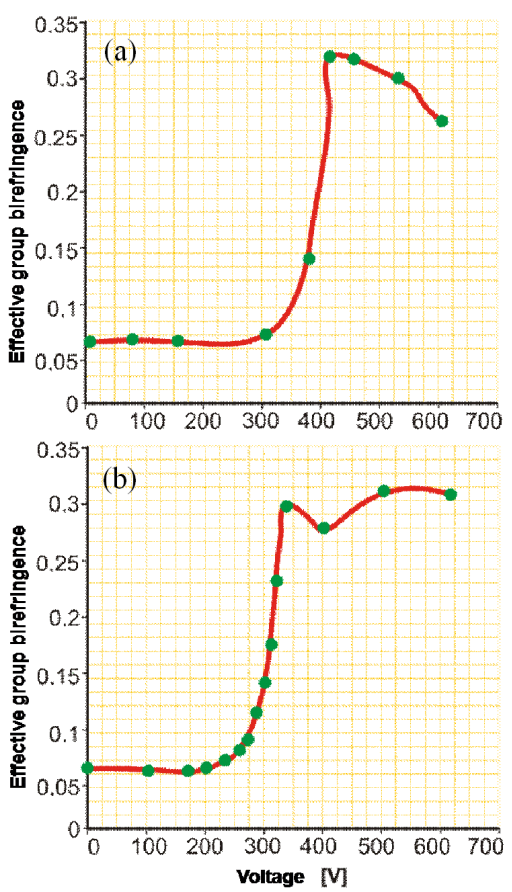

Fig. 8. Effective group birefringence calculated from depolarization based method for (a) horizontally and vertically (b) aligned set of electrodes for laser diode emitting green wavelength.

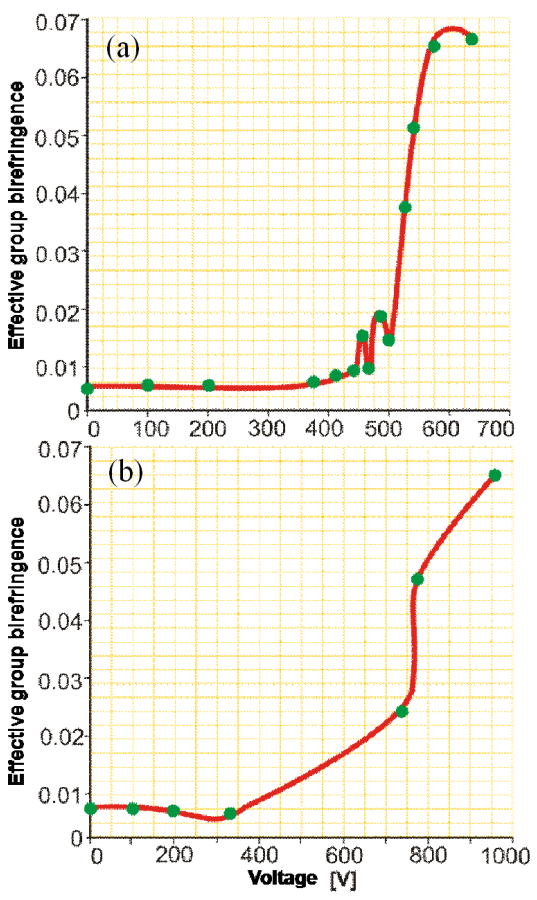

Fig. 9. Effective group birefringence calculated from depolarization based method for (a) horizontally and vertically (b) aligned set of electrodes for laser diode emitting red wavelength. 
increasing the voltage on the function generator we were registering DOP at the fiber output and next we calculated effective group birefringence of the PLCF according to Eq. (17). The results for green laser diode light beam are shown in Fig. 8.

By increasing the voltage between electrodes the effective group birefringence of the PLCF was increasing. The same effect was observed for red laser diode light beam (Fig. 9)

\section{Conclusions}

We have introduced a novel non-interferometric method of calculating effective group birefringence of the birefringent media like PCF infiltrated with molecules of LC, which stands in very good agreement with other interferometric methods. By use of external electric field we are able to control orientation of the molecules of LC placed inside PCF micro-holes and hence to change effective group birefringence. The results presented in this paper will be useful in designing fiber optic depolarizer based on PLCFs.

\section{Acknowledgments}

This work has been supported by the European Union in the framework of European Social Fund through the Warsaw University of Technology Development Programme, and by Polish Ministry of Science and Higher Education under the grant No. 4067/B/T02/2008/34 and the grant No. M 517056535.

\section{References}

[1] T.R. Woliński, S. Ertman, A. Czapla, P. Lesiak, K. Nowecka, A.W. Domański, E. Nowinowski-Kruszelnicki, R. Dąbrowski, J. Wójcik, Meas. Sci. Technol. 18, 3061 (2007)

[2] A.W. Domanski, Optoelectronics Rev. 13, 171 (2005).

[3] A.W. Domański, D. Budaszewski, M. Sierakowski, T.R. Woliński, Optoelectronics Rev. 14, 305 (2006).

[4] D. Budaszewski, A.W. Domański, A. Czapla, S. Ertman, T.R. Woliński, T. Nasiłowski, H. Thienpont, Optoelectronics Rev. 17, 156 (2009).

[5] A.W. Domański, D. Budaszewski, S. Ertman, P. Lesiak, K. Nowecka, T.R. Woliński, Proc. SPIE 6608, 660807 (2007).

[6] T. Pustelny, M. Grabka, Acta Phys. Pol. A 114, A-113 (2008).

[7] D. Budaszewski, R. Cieślak, A.W. Domański, Mol. Cryst. Liquid Cryst. 502, 47 (2008). 